\title{
A Non-Simple and Not Completed History of Quantum Mechanics and Really Long History of Resolving the Problem of Time as a Quantum Observable
}

\author{
Vladislav S. Olkhovsky \\ Institute for Nuclear Research of NASU, Prospect Nauki, Kiev, Ukraine \\ Email: olkhovsky@mail.ru
}

Received 7 July 2014; revised 23 August 2014; accepted 25 September 2014

Copyright (C) 2014 by author and OALib.

This work is licensed under the Creative Commons Attribution International License (CC BY).

http://creativecommons.org/licenses/by/4.0/

(c) (i) Open Access

\begin{abstract}
It shortly described a far from being completed history of quantum mechanics-quantum theory of measurements. Furthermore, there was a relatively recently finished chapter of quantum mechanics which for a long time had not been resolved. This chapter is dedicated to time as a quantum observable, canonically conjugated to energy. And the mathematical reasons for its principal resolving are explained.
\end{abstract}

\section{Keywords}

Quantum Mechanics, Unresolved Problem of the Interpretation of Quantum Theory, Quantum Theory of Measurements, Maximal Hermitian Time Operator for Systems with the Continouous Energy Spectra, Measure (Weight) of Averaging Over Time, Time Operator for Systems with the Discrete Energy Spectra

Subject Areas: Modern Physics, Nuclear Physics, Quantum Mechanics

\section{Introduction}

The problem of the profound interpretation and entire understanding of quantum mechanics (i.e. joint of the fundamental quantum mechanics with quantum theory of measurements) up to now has been actual. Besides, quantum mechanics, the foundations of which had been constructed during the 20th of XX century, a long time (practically till the end of the XX century-the start of XXI century) had not contained the principally resolved chapter of time as a quantum observable canonically conjugated to energy. 


\section{Comments on the Unresolved Problem of the Interpretation and Entire Understanding of Quantum Mechanics}

There is presented a large list of unresolved problems of physics in [1]. Here let turn our attention to one great problem of physics - to the problem of the interpretation and entire understanding of quantum mechanics which is still remained to be topical.

Firstly this problem had arisen evidently during the discussion between N. Bohr with A. Einstein which had been continued a lot of years and had not been finished not only because of the Second World War. Even after the formal acceptance the Copenhagen point of view by the majority of physicians in the probabilistic interpretation of wave function there had not been reached the total consensus between physicians. That discussion is continued by the contemporary physicians, sometimes in rather acute forms.

Many critics of quantum mechanics do not satisfied up to now by the probabilistic nature of its predictions. Moreover, the questions of quantum theory of measurements like the wave-function reduction during measurements and of the paradox EPR (the Einstein, Podolsky, Rosen paradox) are remained to be acute [2]. By the way, the beginning of the solution of time in quantum mechanics brought to one more experimentally observed paradox-the Hartmann effect during the tunneling of particles and photons with its generalizations and extensions [3]-[5] which testifies (as the paradox EPR) on the non-locality of quantum mechanics and quantum electrodynamics with the observable superluminal group velocities of tunneling photons.

Moreover, unlike to classic, non-quantum physics, there is the un-removable indeterminacy on the microscopic level, presented by the Heisenberg indeterminacy relations, by the non-localizability of the not yet unmeasured particles-waves and also by the problem of the quantum-measurement interpretations in the cases when the state of the measured system is formed by an observer. And if the majority of physicians agree with the N. Bohr Copenhagen interpretation of quantum mechanic, a certain part of physicians assume that A. Einstein was right in his statement that quantum theory in the Copenhagen interpretation does not directly describe the reality.

\section{Various Interpretations of Quantum Mechanics}

A number of physicians, beginning from A. Einstein, D. Bohm, Ya. Aharonov etc., do not till now with the probabilistic (Copenhagen) interpretation of quantum mechanics and moreover had constructed alternative versions of their interpretations (see [6]-[10]).

Einstein did never accept quantum mechanics as a "real" and complete theory, fighting all his life for the interpretation which could be compatible with the relativity theory without acceptance the Heisenberg indeterminacy relations. Once he stated: “God doesn't play dice with the world”, skeptically relating to the probabilistic interpretation of quantum mechanics which states that there is no objective reality, other than can be revealed by measurements and observations. And approximately after 1970 it was appeared the interpretation of many worlds or universes (IMW).

\section{Interpretation of Many Worlds (IMW) and Some Other Interpretations in Quantum Mechanics}

There is assumed the existence of parallel universes in this interpretation of quantum mechanics (and quantum cosmology). In every from them the same nature laws and the same physical constants act, but all of them are found in various states. IMW does not contain the undetermined collapse of wave function which is connected with the measurement in the Copenhagen interpretation. The ideas of IMW were appeared in the dissertation of G. Everett but the term IMW was proposed by B. S. M de Witt who was developed this idea and then a number of other authors were participated in the development of this interpretation [11]-[13]. Peculiar interesting version of IMW with the inclusion of the human conscience was put forced by Russian physicist M. B. Menskii [14].

There are two main statements in various IMW versions: The fist statement is the existence of wave function of the whole Universe which is described by the Schrödinger equation but without any undetermined collapse. The second statement is the following: every state of the Universe the quantum superposition of several (or, may be, of infinite number of) states of equal parallel universes which do not interact one with each other.

Some opponents of IMW reject it because the infinite number of universes seems to them the very strong violation of the principle "Ockham razor": "The number of essences should not be multiplied above the necessity”. 
However in evaluation of physical theories it is appropriately to assume that the number of physical theories should not be multiplied above the necessity also (namely such version of the Ockham razor was applied in the past). And in this sense IMW is the most economic theory. Really, it contains all the laws of quantum mechanics but without the problematic postulate of collapse. Also it is very difficult to understand or to be accustomed to the thought that every microscopic measurement can transfer a researcher in the new universe.

The main argument for the acceptance of IMW is the absence in it of the collapse of wave function. Besides that, also the experimental testimonies in the favor of collapse and against IMW are absent. And there is no need to assume that God does not play dice: IMW is the deterministic theory of physical Universe and it explains why the world seems to be un-deterministic for the human observer.

IMW is not the most popular interpretation of quantum theory between physicians but its popularity apparently increases. Most of all the supporters of IMW are in the quantum cosmology and in quantum computation. In quantum cosmology it is possible to research the Universe, avoiding the difficulty of the standard interpretation with the external observer. In quantum computation the key advantage is that the parallel computations are realized by the same computer; it enters in the main picture of IMW. Moreover, the advantage of IMW in quantum mechanics is that it permits to consider quantum mechanics as the complete and consistent physical theory which is compatible with all known experimental results. And also starting from the elegant conception of the de-coherence proposed in 1970 by D. Zeh, it is possible to explain that the various branches of the unique wave function, describing these worlds, are oscillating in time with phase shift and therefore each for other as if do not exist [15].

At the same time the Polish physician J. A. Janik proposed such simple generalization of the Copenhagen interpretation which is consistent with the doctrine of the Intelligent Design: If the Creator created quantum mechanics, then he had realized the own Design also as the constant Observer of micro-events [16].

\section{Instead of Conclusion on the Final Interpretation of Quantum Mechanics}

Now the problem of the final interpretation of quantum mechanics and quantum measurement theory is still far from the total consensus.

And till now it is often stated that quantum mechanics shows that during time interval between our measurements there is no objective reality which could not depend from us as from observers. Some researchers use, instead of the description of the quantum world as the created by an observer reality, more modest term of reality belonged under the influence of an observer (see [17]).

\section{Time as a Quantum Observable Canonically Conjugate to Energy (Historical Introduction)}

Really, during near ninety years after the remark of Pauli (see, for example, [18], where he rejected time as a quantum observable because of the absence of time self-adjoint operator) it is known that time cannot be really represented by a self-adjoint operator, with the possible exception of special abstract systems (such as an electrically charged particle in an infinite uniform electric field and a system with the limited from both below and above energy spectrum). This fact results to be in contrast with the known circumstance that time, as well as space, in some cases plays the role just of a parameter, while in some other cases is a physical observable which ought to be represented by an operator. The same situation had to be faced also in quantum electrodynamics and, more in general, in relativistic quantum field theory (see, for instance, [3]-[5] [19]-[22]). So, the fundamental quantum mechanics a long time (near 90 years after [18]) appeared to be incomplete: time was not a quantum observable, time really was not used as a quantum observable, time was used as physically really a classic parameter. There was not exact derivation of time-energy indeterminacy relation as for coordinate impulse equations and there were long discussions between physicists about its understanding.

The most important foundation of the results [3]-[5] [19]-[21] was the mathematic Naimark theorem from [23]. This Naimark theorem states that the non-orthogonal spectral decomposition $E(\lambda)$ of a hermitian operator $H$ is of the Carleman type [24] (which is unique for the maximal hermitian operator), i.e. it can be approximated by a succession of the self-adjoint operators, the spectral functions of which do weakly converge to the spectral function $E(\lambda)$ of the operator $H$.

Namely, by exploiting that mathematic Naimark theorem, it has been shown in [3]-[5] [19]-[21] that, for systems with continuous energy spectra, time can be introduced as a quantum-mechanical observable, canonically 
conjugate to energy. More precisely, the time operator resulted to be maximal hermitian, even if not self-adjoint. Here we briefly describe a self-consistent quantum approach to time, canonically conjugate to energy.

\section{Time as a Quantum Observable and General Definitions of Mean Times and Mean Durations of Quantum Processes for Systems with Continuous Energy Spectra}

From [3]-[5] [19]-[21] for systems with continuous energy spectra, the following simple operator, canonically conjugate to energy, can be introduced for time:

$$
\grave{i}=\left\{\begin{array}{l}
t \text { in time }(t-) \text { representation } \\
-i \hbar \partial / \partial E \text { in energy }(E-) \text { representation. }
\end{array}\right.
$$

which is not self-adjoint, but is hermitian, and acts on square-integrable space-time wave packets in Representation (1a), and on their Fourier-transforms in Representation (1b), once the point $E=0$ is eliminated (i.e., once one deals only with moving packets, i.e., excludes any non-moving back tails, as well as, of course, the zero flux cases). Such a condition is enough for operator (1a), (1b) to be a "maximal hermitian" operator, according to the terminology of [24]. The elimination of the point $E=0$ is not restrictive since the "rest" states with the zero velocity, the wave packets with non-moving rear tails, and the wave packets with zero flux are unobservable.

Operator (1b) is defined as acting on the space $P$ of the continuous, differentiable, square-integrable functions $f(E)$ that satisfy the conditions

$$
\int_{0}^{\infty}|f(E)|^{2} \mathrm{~d} E<\infty, \quad \int_{0}^{\infty}|\partial f(E) / \partial E|^{2} \mathrm{~d} E<\infty, \quad \int_{0}^{\infty}|f(E)|^{2} E^{2} \mathrm{~d} E<\infty
$$

and

$$
f(0)=0 \text {. }
$$

The space $P$ is dense in the Hilbert space of $L^{2}$ functions defined (only) over the semi-axis $0 \leq E<\infty$. Obviously, the operator $(1 \mathrm{a}, \mathrm{b})$ is hermitian, i.e. the relation $\left(f_{1}, \hat{t} f\right)=((\hat{t} f), f)$ holds, only if all squareintegrable functions $f(E)$ in the space on which it is defined vanish for $E=0$.

Operator $\hat{t}$ has no hermitian extension because otherwise one could find at least one function $f_{0}(E)$ which satisfies the condition $f_{0}(0) \neq 0$ but that is inconsistent with the propriety of being hermitian. So, according to [25], $\hat{t}$ is a maximal hermitian operator.

Essentially because of these reasons, earlier Pauli (see, for instance, [18]) rejected the use of a time operator: and this had the result of practically stopping studies on this subject for near 40 - 50 years (because the Naimark work [23] and more old Carleman work [24] were unknown during a long time for physicians). However, as far back as in [26] von Neumann had claimed that considering in quantum mechanics only self-adjoint operators could be too restrictive. And in [26] he himself brought such example with a particle, freely moving in the space with the semi-axis $x \quad(0 \leq x<\infty)$, which is limited by the rigid wall along the plane $x=0$. Operator of the $x$ component for such particle $\hat{p}_{x}=-i \hbar \frac{\partial}{\partial x}$ is defined as acting on the space of the continuous, differentiable, square-integrable functions $f(x)$ that satisfy the conditions

$$
\int_{0}^{\infty}|f(x)|^{2} \mathrm{~d} x<\infty, \quad \int_{0}^{\infty}|\partial f(x) / \partial x|^{2} \mathrm{~d} x<\infty, \quad \int_{0}^{\infty}|f(x)|^{2} x^{2} \mathrm{~d} x<\infty
$$

and

$$
f(0)=0 \text {. }
$$

This space of functions $Q$ is dense in the Hilbert space of $L^{2}$ functions defined (only) over the semi-axis $0 \leq x<\infty$. Therefore operator $\hat{p}_{x}=-i \hbar \frac{\partial}{\partial x}$ has the same mathematical properties as operator $\hat{t}$ ((1a), (1b)) and, consequently, is non self-adjoint but maximal hermitian operator. Nevertheless, it continues to remain an observable with evident physical sense. The same properties has operator of the radial impulse $\hat{p}_{r}=-i \hbar \frac{\partial}{\partial r}+\frac{1}{r}$ 
$(0<r<\infty)$.

Long time ago it was noted that in the case of the hypotetical quantum systems with the continuous energy spectra which are limited from below and from above $\left(E_{\min }<E<E_{\max }\right)$ time operator ((1a), (1b)) will be real self-adjoint operator and has the discrete time spectrum with the "time quant" $t=\hbar / d$ where $d=\left|E_{\max }-E_{\min }\right|$.

In order to consider time as an observable in quantum mechanics and to define the observable mean times and durations, one needs to introduce not only the time operator, but also, in a self-consistent way, the measure (or weight) of averaging over time. In the simple one-dimensional (1D) and one-directional motion such measure (weight) can be obtained by the simple quantity:

$$
W(x, t) \mathrm{d} t=\frac{j(x, t) \mathrm{d} t}{\int_{-\infty}^{\infty} j(x, t) \mathrm{d} t}
$$

where the probabilistic interpretation of $j(x, t)$ (namely in time) corresponds to the flux probability density of a particle passing through point $x$ at time $t$ (more precisely, passing through $x$ during a unit time interval, centered at $t$ ), when travelling in the positive $x$-direction. Such a measure had not been postulated, but is just a direct consequence of the well-known probabilistic (spatial) interpretation of $\rho(x, t)$ and of the continuity relation

$$
\partial \rho(x, t) / \partial t+\operatorname{divj}(x, t)=0
$$

for particle motion in the field of any hamiltonian in the description of the 1D Schroedinger equation. Quantity $\rho(x, t)$ is the probability of finding a moving particle inside a unit space interval, centered at point $x$, at time $t$. The probability density $\rho(x, t)$ and the flux probability-density $j(x, t)$ are related with the wave function $\Psi(x, t)$ by the usual definitions $\rho(x, t)=|\Psi(x, t)|^{2}$ and $j(x, t)=\operatorname{Re}[\Psi(x, t)(\hbar / i \mu) \partial \Psi(x, t) / \partial x]$. The measure (4) was firstly investigated in [3]-[5].

When the flux density $j(x, t)$ changes its sign, the quantity $W(x, t) \mathrm{d} t$ is no longer positive definite and it acquires a physical meaning of a probability density only during those partial time-intervals in which the flux density $j(x, t)$ does keep its sign. Therefore, let us introduce the two measures, by separating the positive and the negative flux-direction values (i.e., flux signs):

$$
W_{ \pm}(x, t) \mathrm{d} t \frac{j_{ \pm}(x, t) \mathrm{d} t}{\int_{-\infty}^{\infty} j_{ \pm}(x, t) \mathrm{d} t}
$$

with $j_{ \pm}(x, t)=j(x, t) \Theta( \pm j)$ where $\Theta(z)$ is the Heaviside step function. It had been made firstly in [3]-[5] [20]. Actually, one can rewrite the continuity relation (5) for those time intervals, for which $j=j_{+}$or $j=j_{-}$ as follows:

$$
\frac{\partial \rho_{>}(x, t)}{\partial t}=-\frac{\partial j_{+}(x, t)}{\partial x} \text { and } \frac{\partial \rho_{<}(x, t)}{\partial t}=-\frac{\partial j_{-}(x, t)}{\partial x}
$$

respectively.

Then, one can eventually define the mean value $\langle t(x)\rangle$ of the time $t$ at which a particle passes through position $x$ (when travelling in only one positive $x$-direction), and $\left\langle t_{ \pm}(x)\right\rangle$ of the time $t$ at which a particle passes through position $x$, when travelling in the positive or negative direction, respectively :

$$
\langle t(x)\rangle=\frac{\int_{-\infty}^{\infty} t j(x, t) \mathrm{d} t}{\int_{-\infty}^{\infty} j(x, t) \mathrm{d} t}=\frac{\int_{0}^{\infty} \mathrm{d} E \frac{1}{2}\left[G^{*}(x, E) \hat{t} v G(x, E)+v G^{*}(x, E) \hat{t} G(x, E)\right]}{\int_{0}^{\infty} \mathrm{d} E v|G(x, E)|^{2}}
$$

where $G(x, E)$ is the Fourier-transform of the moving one-dimensional (1D) wave packet

$$
\Psi(x, t)=G(x, E) \exp (-i E t / \hbar) \mathrm{d} E=g(E) \varphi(x, E) \exp (-i E t / \hbar) \mathrm{d} E
$$


when going on from the time representation to the energy one,

$$
\left\langle t_{ \pm}(x)\right\rangle=\frac{\int_{-\infty}^{\infty} t j_{ \pm}(x, t) \mathrm{d} t}{\int_{-\infty}^{\infty} j_{ \pm}(x, t) \mathrm{d} t},
$$

and also the mean durations of particle 1D transmission from $x_{i}$ to $x_{f}>x_{i}$ and 1D particle reflection from the region $\left(x_{i}, \infty\right)$ into $x_{f} \leq x_{i}$ :

$$
\left\langle t_{T}\left(x_{i}, x_{f}\right)\right\rangle=\left\langle t_{+}\left(x_{f}\right)\right\rangle-\left\langle t_{+}\left(x_{i}\right)\right\rangle \text { and }\left\langle t_{R}\left(x_{i}, x_{f}\right)\right\rangle=\left\langle t_{-}\left(x_{f}\right)\right\rangle-\left\langle t_{+}\left(x_{i}\right)\right\rangle
$$

respectively. Of course, it is possible to pass in Equation (7b) also to integrals $\int_{0}^{\infty} \mathrm{d} E \cdots$, similarly to (7a) by using the unique Fourier (Laplace)-transformations and the energy expansion of $j_{ \pm}(x, t)=j(x, t) \theta( \pm j)$, but it is evident that they result to be rather bulky. The three-dimensional case can be similarly analyzed [5] [19].

\section{Time-Energy Uncertainty Relation for Systems with the Continuous Energy Spectra}

Now, one can see that two canonically conjugate operators, the time operator (1) and the energy operator

$$
\hat{E}=\left\{\begin{array}{l}
E \text { in the energy }(E-) \text { representation, } \\
\text { i } \hbar \frac{\partial}{\partial t} \text { in the time }(t-) \text { representation. }
\end{array}\right.
$$

satisfy the typical commutation relation

$$
[\hat{E}, \hat{t}]=i \hbar
$$

Although up to now according to the Stone and von Neumann theorem [27] the relation (10) has been interpreted as holding only for the pair of the self-adjoint canonically conjugate operators, in both representations, and it was not directly generalized for maximal hermitian operators, the difficulty of such direct generalization has in fact been by-passed by introducing $\hat{t}$ with the help of the single-valued Fourier (Laplace)-transformation from the $t$-axis $(-\infty<t<\infty)$ to the $E$-semi-axis $(0<E<\infty)$ and by utilizing the peculiar mathematical properties of maximal hermitian operators, described in detail, e.g., in [5] [19]-[21].

Actually, from Equation (10) the uncertainty relation

$$
\Delta E \Delta t \geq \hbar / 2
$$

(where the standard deviations are $\Delta a=\sqrt{ } \mathrm{D} a$, quantity $\mathrm{D} a$ being the variance $\mathrm{D} a=\left\langle a^{2}\right\rangle-\langle a\rangle^{2}$; and $a=E$, $t$, while $\langle\cdots\rangle$ denotes an average over $t$ by the measures $W(x, t) \mathrm{d} t$ or $W_{ \pm}(x, t) \mathrm{d} t$ in the $t$-representation or an average over $E$ similar to the right-hand-part of (7a) and (8) in the $E$-representation) was derived by the simple generalizing of the similar procedures which are standard in the case of self-adjoint canonically conjugate quantities. Moreover, relation (10) satisfies the Dirac “correspondence principle”, since the classical Poisson brackets $\left\{q_{0}, p_{0}\right\}$, with $q_{0}=t$ and $p_{0}=-E$, are equal to unity [28]. In [5] [19]-[21] it was also shown that the differences between the mean times at which a wave-packet passes through a pair of points obey the Ehrenfest correspondence principle; in other words, the Ehrenfest theorem was suitably generalized.

After what precedes, one can state that, for systems with continuous energy spectra, the mathematical properties of the maximal hermitian operators, like $\hat{t}$ in Equation (1), are sufficient for considering them as quantum observables: Namely, the uniqueness of the "spectral decomposition" (also called spectral function) for operators $\hat{t}$, as well as for $\hat{t}^{n} \quad(n>1)$ guarantees (although such an expansion is not orthogonal) the equivalence of the mean values of any analytic functions of time, evaluated either in the $t$ - or in the $E$-representations. In other words, the existence of this expansion is equivalent to a completeness relation for the (formal) eigen functions of $\hat{t}^{n} \quad(n>1)$, corresponding with any accuracy to real eigen values of the continuous spectrum; such eigen functions belonging to the space of the square-integrable functions of the energy $E$ with the boundary conditions (2)-(3). 
From this point of view, there is no practical mathematical difference between self-adjoint and maximal hermitian operators for systems with continuous energy spectra. Let us underline that the mathematical properties of $\hat{t}^{n} \quad(n>1)$ are quite enough for considering time as a quantum-mechanical observable (like for energy, momentum, spatial coordinates, ...) without having to introduce any new physical postulates. Only the mathematical base of quantum mechanics becomes broader than earlier.

\section{Time as an Observable and Time-Energy Uncertainty Relation for Quantum-Mechanical Systems with Discrete Energy Spectra}

For systems with discrete energy spectra it is natural (following [5] [20] [21]) to introduce wave packets of the form

$$
\Psi(x, t)=\sum_{n=0}^{m} g_{n} \varphi_{n}(x) \exp \left[-i\left(\varepsilon_{n}-\varepsilon_{0}\right) t / \hbar\right]
$$

(where $\varphi_{n}(x)$ are orthogonal and normalized wave functions of system bound states which satisfy equation $\hat{H} \varphi_{n}(x)=\varepsilon_{n} \varphi_{n}(x), \hat{H}$ being the system Hamiltonian; $\sum_{n=0}\left|g_{n}\right|^{2}=1$. Here we factually omitted a non-significant phase factor $\exp \left(-i \varepsilon_{0} t / \hbar\right)$ as being general for all terms of the sum $\sum_{n=0}$ ) for describing the evolution of systems in the regions of the purely discrete spectrum. Without limiting the generality, we choose moment $t=0$ as an initial time instant.

Firstly, we shall consider those systems, whose energy levels are spaced with distances for which the maximal common divisor exists in fact. Examples of such systems are harmonic oscillator, particle in a rigid box and spherical spinning top. For these systems the wave packet (12) is a periodic function of time with the period (Poincaré cycle time) $T=2 p \hbar / D, D$ being the maximal common divisor of distances between system energy level.

In the $t$-representation the relevant energy operator $\hat{H}$ is a self-adjoint operator acting in the space of periodical functions whereas the function $t \psi(t)$ does not belong to the same space. In the space of periodical functions the time operator $\hat{t}$, even in the eigen representation, has to be also a periodical function of time $t$. This situation is quite similar to the case of azimuth angle $\varphi$ which is canonically conjugated to angular momentum $\hat{L}_{z}$ (see, for instance, [29] [30]). Utilizing the example and result from [31], let us choose, instead of $t$, a periodical function

$$
\hat{t}=t-T \sum_{n=0}^{\infty} \Theta(t-[2 n+1] T / 2)+T \sum_{n=0}^{\infty} \Theta(-t-[2 n+1] T / 2),
$$

which is the so-called saw-function of $t$ (see Figure 1).

This choice is convenient because the periodical function of time operator (13) is linear function (one-directional) within each Poincaré interval, i.e. time conserves its current and its usual meaning of an order parameter for the system evolution.

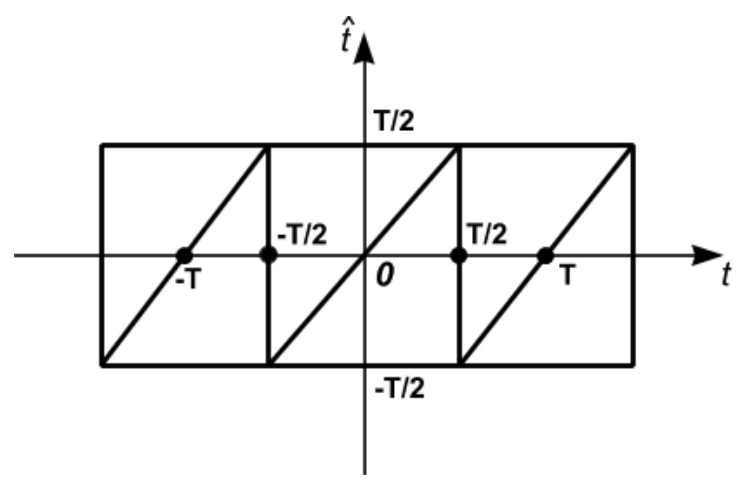

Figure 1. The periodical saw-tooth function for time operator for the case of (13). 
The commutation relation of the self-adjoint energy and time operators acquires in this case (discrete energies and periodical functions) the form:

$$
[\hat{E}, \hat{t}]=i \hbar\left\{1-T \sum_{n=0}^{\infty} \delta(t-[2 n+1] T)\right\}
$$

Let us recall (see, e.g. [31]) that a generalized form of uncertainty relation holds

$$
(\Delta A)^{2} \cdot(\Delta B)^{2} \geq \hbar^{2}[\langle N\rangle]^{2}
$$

for two self-adjoint operators $\hat{A}$ and $\hat{B}$, canonically conjugate each to other by the commutator

$$
[\hat{A}, \hat{B}]=i \hbar \hat{N} \text {, }
$$

$\hat{N}$ being a third self-adjoint operator. One can easily obtain

$$
(\Delta E)^{2} \cdot(\Delta t)^{2} \geq \hbar^{2}\left[1-\frac{T|\psi(T / 2+\gamma)|^{2}}{\int_{-T / 2}^{+T / 2}|\psi(t)|^{2} \mathrm{~d} t}\right],
$$

where the parameter $\gamma$ (with an arbitrary value between $-T / 2$ and $+T / 2$ ) is introduced for the univocality of calculating the integral on right part of (17) over $\mathrm{d} t$ in the limits from $-T / 2$ to $+T / 2$, just similarly to the procedure introduced in [29] (see also [31]).

From (17) it follows that when $\Delta E \rightarrow 0$ (i.e. when $\left|g_{n}\right| \rightarrow \delta_{n n}$ ) the right part of (17) tends to zero since $|\psi(t)|^{2}$ tends to a constant. In this case the distribution of time instants of wave-packet passing through point $x$ in the limits of one Poincaré cycle becomes uniform. When $\Delta E \gg D$ and $|\psi(T+\gamma)| \ll T \int_{-T / 2}^{T / 2}|\psi(t)|^{2} \mathrm{~d} t$, the periodicity condition may be inessential for $\Delta t \ll T$, i.e. (17) passes to uncertainty relation (7), which is just the same one as for systems with continuous spectra.

In principle, one can obtain the expression for time operator (13) also in energy representation.

In general cases, for excited states of nuclei, atoms and molecules, level distances in discrete spectra have not strictly defined the maximal common divisor and hence they have not the strictly defined time of the Poincaré cycle. And also there is no strictly defined passage from the discrete part of the spectrum to the continuous part. Nevertheless, even for those systems one can introduce an approximate description (and with any desired degree of the accuracy within the chosen maximal limit of the level width, let us say, $\gamma_{\lim }$ ) by quasi-cycles with quasiperiodical evolution and for sufficiently long intervals of time the motion inside such systems (however, less than $\hbar / \gamma_{\text {lim }}$ ) one can consider as a periodical motion also with any desired accuracy. For them one can choose (define) a time of the Poincare' cycle with any desired accuracy, including in one cycle as many quasi-cycles as it is necessary for demanded accuracy. Then, with the same accuracy the quasi-self-adjoint time operator (13) can be introduced and all time characteristics can be defined.

In the degenerate case when at the state (12) the sum $\sum_{n=0}^{\infty}$ contains only one term ( $g_{n} \rightarrow \delta_{n n}$ ), the evolution is absent and the time of the Poincare' cycle is equal formally to infinity.

If a system has both (continuous and discrete) regions of the energy spectrum, one can easily use the forms (1) for the continuous energy spectrum and the forms (13) for the discrete energy spectrum.

\section{Time as an Observable Canonically Conjugated to Energy in Quantum Electrodynamics and Quantum Field Theory}

It was shown in [5] [20] [21] [32] [33] that energy is a quantum observable in quantum electrodynamics and for the Klein-Gordon equation. For the Klein-Gordon equation [33] the continuity relation will be also (5) where 
after passage into the impulse space for particles $\rho=|\psi(x, t)|^{2}=\int_{+} \frac{\mathrm{d}^{3} k}{k_{0}}|\Phi(k)|^{2}, \frac{\mathrm{d}^{3} k}{k_{0}}$ is an element of the Lorentz-invariant weight on the hyperboloid $k^{2}=\mu_{0}^{2}$, and the probability flux density will be equal $j=(2 m)^{-1}\left(\Psi^{*} p \Psi-\Psi p \Psi^{*}\right)$, as in non-relativistic quantum mechanics. Then the weight of averaging over time for a 1-D moving particle will be (4) or (4a), as in non-relativistic case.

In the case of the Dirac equation [33] the continuity equation will be also (5), where

$$
\rho=|\psi(x, t)|^{2}, \quad j_{k}=c \Psi^{*}(x, t) a_{k} \Psi(x, t), \quad a_{k}=\left(\begin{array}{cc}
0 & \sigma_{k} \\
\sigma_{k} & 0
\end{array}\right), \quad \sigma_{1}=\left(\begin{array}{cc}
0 & 1 \\
1 & 0
\end{array}\right), \quad \sigma_{2}=\left(\begin{array}{cc}
0 & -i \\
i & 0
\end{array}\right), \quad \sigma_{2}=\left(\begin{array}{cc}
1 & 0 \\
0 & -1
\end{array}\right) .
$$

and then the weight of averaging over time for a 1-D moving particle will be also (4) or (4a).

\section{On the Impulse Representation for Time Operator}

It was demonstrated in [34] that in the case of energy continuous spectrum it is possible to use in (8) instead of energy $(E-)$ representation with $0<E<\infty$ also impulse $(k-)$ representation with such advantage that impulse values $k$ occupy total infinite axis from $-\infty$ till $\infty$

$$
\Psi(x, t)=\int_{-\infty}^{\infty} \mathrm{d} k g(E) \varphi(x, E) \exp (-i E t / \hbar),
$$

where $E=\hbar^{2} k^{2} / 2 m, \quad k \neq 0$. In such case time operator ((1a), (1b)) is already formally self-adjoint in acting on the impulse eigen function $\mathrm{e}^{i k x}$ along the axis $-\infty<k<\infty$ with regard to the boundary conditions

$$
\left[\frac{\mathrm{d}^{n} g(k)}{\mathrm{d} k^{n}}\right]_{k=-\infty}=\left[\frac{\mathrm{d}^{n} g(k)}{\mathrm{d} k^{n}}\right]_{k=\infty}=0, \quad n=0,1,2, \cdots,
$$

with the exception of one point $k=0$, elimination of which has the physical sense of non-observation of the "rest" (absence of motion) which is mathematically inessential. And it is really one more argument that time is an observable as any other quantity, to which corresponds self-adjoint operator.

Let us compare the choice (18) with (8) and rewrite (18) concretely in the form:

$$
\begin{aligned}
\Psi(x, t)= & \int_{0}^{\infty} \mathrm{d} E(E)^{-1 / 2} g\left(\frac{(2 m E)^{1 / 2}}{\hbar}\right) \varphi\left(x, \frac{(2 m E)^{1 / 2}}{\hbar}\right) \exp \left(-\frac{i E t}{\hbar}\right) \\
& +\int_{0}^{\infty} \mathrm{d} E(E)^{-1 / 2} g\left(-\frac{(2 m E)^{1 / 2}}{\hbar}\right) \varphi\left(x-\frac{(2 m E)^{1 / 2}}{\hbar}\right) \exp \left(-\frac{i E t}{\hbar}\right) .
\end{aligned}
$$

If one introduce the weight function

$$
\tilde{g}(E)=\left(\frac{m}{2 E \hbar^{2}}\right)^{1 / 4}\left[\begin{array}{c}
g\left(\frac{(2 m E)^{1 / 2}}{\hbar}\right) \\
g\left(-\frac{(2 m E)^{1 / 2}}{\hbar}\right)
\end{array}\right]
$$

as a "two-dimensional" vector, then

$$
\int_{-\infty}^{\infty}|\Psi(x, t)|^{2} \mathrm{~d} x=\int_{0}^{\infty}|\tilde{g}(E)|^{2} \mathrm{~d} E<\infty,
$$

where the Euclidean norm of two-dimensional vector $|\tilde{g}(E)|^{2}=\tilde{g}^{*}(E) \cdot \tilde{g}(E)>0$.

If wave packet (18) is one-directional and $g(k) g(k) \Theta(k)$, then integral $\int_{-\infty}^{\infty} \mathrm{d} k$ passes into integral $\int_{0}^{\infty} \mathrm{d} k$, and two-dimensional vector passes into scalar. In this case it is possible to replace the bounded conditions (2) and (3) by the conditions of the same form with replacing $E \rightarrow k$. By the way, in the impulse representation 
time can be easily recognized as a quantum observable.

\section{Introduction of "Hamiltonian" Analogy for Time Operator: New Hamiltonian Approach for Time Operator}

It is well-known in the quantum theory that there is the correspondence between energy $E$ and two operatorsoperator $i \hbar \frac{\partial}{\partial t}$ in $t$-representation and the Hamiltonian operator. Duality of these operators one can easily see from the Schroedinger equation $\hat{H} \Psi=i \hbar \frac{\partial}{\partial t}$. Similar duality must take place also for time in quantum theory: besides the general form ((1a),(1b)), which takes place for every physical system (with continuous energy spectrum), one can express time operator also in terms of coordinate and impulse operators, using commutation relation between Hamiltonian and time operator. And so, if one makes the substitution

$$
\left\{\begin{array}{c}
\hat{E} \rightarrow \hat{H}\left(\hat{p}_{x}, \hat{x}, \cdots\right) \\
\hat{t} \rightarrow \hat{T}\left(\hat{p}_{x}, \hat{x}, \cdots\right)
\end{array}\right.
$$

then one obtains

$$
[\hat{H}, \hat{T}]=i \hbar,
$$

which is similar to (3). Commutation relation (21) can be used in order to find $\hat{T}\left(\hat{p}_{x}, \hat{x}, \cdots\right)$ for any concrete physical system with the known Hamiltonian $\hat{H}\left(\hat{p}_{x}, \hat{x}, \cdots\right)$ (see, for instance, [35]-[37]). However, one can note a curious detail which in some cases induced a confusion with the sign in (1b) and in right part of (21). Choosing the coordinate or the impulse representation for $\hat{H}\left(\hat{p}_{x}, \hat{x}, \cdots\right)$ and $\hat{T}\left(\hat{p}_{x}, \hat{x}, \cdots\right)$, we do not changed the formal expression for Hamiltonian $\hat{H}\left(\hat{p}_{x}, \hat{x}, \cdots\right)$, but we can change the sign in the formal expression for time operator $\hat{T}\left(\hat{p}_{x}, \hat{x}, \cdots\right)$. One can easily see that for free particle:

$$
\hat{H}=\left\{\begin{array}{l}
\hat{p}_{x}^{2} / 2 m, \hat{p}_{x}=-i \hbar \frac{\partial}{\partial x} \text { in coordinate representation, } \\
\hat{p}_{x}^{2} / 2 m \text { in impulse representation. }
\end{array}\right.
$$

and

$$
\hat{T}=\left\{\begin{array}{l}
\frac{m}{2}\left[\hat{p}_{x}^{-1} x+x \hat{p}_{x}^{-1}\right], \hat{p}_{x}^{-1}=\frac{i}{\hbar} \int \mathrm{d} x \cdots \text { in coordinate representation, } \\
\frac{-m}{2}\left[p_{x}^{-1} \hat{x}+\hat{x} p_{x}^{-1}\right], \hat{x}=i \hbar \frac{\partial}{\partial p_{x}} \text { in impulse representation. }
\end{array}\right.
$$

(in the symmetric form). By the way, Formula (26b) is equivalent to $-i \hbar \frac{\partial}{\partial E}$ because $E=p_{x}^{2} / 2 m$. For the plane wave $\exp (i k x)$, in the both Representations (26a) and (26b), we obtain the same result:

$$
\hat{T} \exp (i k x)=\frac{x}{v} \exp (i k x),
$$

where $\frac{x}{v}$ is time of free motion with the velocity $v$ along the distance $x$.

Introducing "hamiltonian analogy" for time operator, one can finish the formal completion of time as a quantum observable and start to analyze the results of time analysis of quantum processes.

\section{The Results and Remained Problems of Time Analysis of Quantum Processes, Basing on the Application of Time as a Quantum Observable}

Time analysis of quantum processes had been developed in [3] [20] [21] [38]-[43]. 


\subsection{Time Analysis of Tunneling Processes Permitted to Obtain the Following Results}

1) It was established in [3]-[5] that all previously known the partial definitions and receipts of the definitions appeared in the limits of the general Olkhovsky-Recami (O-R) definition as simple partial of the mean tunneling time or the mean square fluctuation in the tunneling-time distribution (or passing in them under special idealized boundary conditions). In certain articles [44] O-R approach was recognized the most self-consistent approach in the limits of standard quantum mechanics (in the Copenhagen interpretation).

2) There is an infinite series of the multiple internal reflections of evanescent and anti-evanescent waves between entrance and exit barrier walls [45].

3) The Hartmann (or Hartmann-Fletcher) "saturation” effect was revealed and analyzed (see, for ex., reviews [3]-[5] [20] [21]). It consists in the independence of tunneling times from the width of sufficiently wide barriers both for total exit (after tunneling) peaks of wave packets and for the peak of every exit packet during all multiple internal reflections. For total exit outwards peaks from the barrier this effect was experimentally confirmed by the tunneling microwave photons in [46]-[53]. They called a series of discussions on the relativistic causality although it is well known clear that the velocity of the wave front (clearly defined for pulses with the sharply increasing forward edge) cannot exceed the light velocity $\boldsymbol{c}$ in vacuum. These discussions had not finished by the consensus, and one from points of view consists it the consequence of the barrier non-locality. There were revealed theoretically the strong violations of the Hartmann effect for the wave packets with the large impulse spreads-with the decreasing tunneling times for increasing barrier width (sometimes even till the negative values) [54].

4) It was theoretically shown in [4] [5] [21] that un-stationary fluxes composed from only evanescent or only anti-evanescent waves separately describe the oscillations originating in all space inside the barrier. It is true for both non-relativistic particles and photons. And it signifies the manifestation of (a) the barrier influence as a whole on the tunneling process for particles and photons and consequently (b) on the non-locality in behavior of tunneling particles and photons inside the barrier [4] [21] [32]. It is curious that it is possible to describe such wave packets by various mathematical methods (passage to virtual (imaginary) impulses, use the Fourier expansion, the instanton approach on the imaginary axis of time) as propagating wave packets in spaces with unusual metrics.

5) Although there is no direct limit passage to classic mechanics for tunneling under the potential barrier particles, nevertheless there are existing non-zero probability density and non-zero probability flux density for wave packets inside the barriers and, moreover, for the motion of wave packets as the super-positions of evanescent and anti-evanescent waves there is evidently direct classical limit: let us record similar motion in time of wave packets as the super-positions of evanescent and anti-evanescent waves in classical optics and classic acoustics [3]-[5] [52] [53].

\subsection{Time Analysis of Nuclear Processes Permitted to Obtain the Following Results}

1) It was developed space-time analysis of nucleon-nucleus scattering cross sections and durations at the region of one resonance, distorted by the non-resonant background, or two overlapped resonances. It was shown that sometimes here arises the time advance instead of time delay in the center-of-mass system, but in the laboratory system there is no advance and only delay [54]-[60].

2) For not very heavy projectiles with projectile energies above 1 - $10 \mathrm{Gev} / \mathrm{nucl}$ there had been observed structure-less, exponentially decaying energy spectra, often accompanied by slight oscillations, throughout the studied range of projectiles and projectile energies, targets and registered final fragments. The inclusive crosssection energy behavior is described by the formula

$$
\sigma=\left|f^{1}\right|^{2} \exp \left(-E \tau_{1} / \hbar\right)+\left|f^{2}\right|^{2} \exp \left(-E \tau_{2} / \hbar\right)+2 \operatorname{Re}\left\{f^{1} f^{2} * \exp \left[i E\left(\tau_{1}-\tau_{2}\right) / \hbar-E\left(\tau_{1}+\tau_{2}\right) / 2 \hbar\right]\right\}
$$

where $\tau_{1,2}$ and $t_{1,2}$ determine the exponential dependence on energy for the correspondent cross section. This corresponds to appearing 2 interfering time resonances (or explosions). It was shown that the origin of phenomena of time-resonances rises from the strict self-consistent time analysis of the compound nuclei at the range of very dense and strongly overlapped energy resonances [61].

3) It was developed space-time analysis of the interference between different mechanisms with three particles (or nuclei) in the final channel [62]. 


\subsection{The First Results of Time Analysis of Nuclear Decays in Astrophysics and Geophysics Are Exposed in [63]-[65]}

where it was initiated the reconsidering of the evaluations for the age of stars and planets, taking into account the physical processes going on inside the large matter masses in stars and in the surface planet layers.

\subsubsection{The Remained Problems}

1) Now it remains to finish the description of 1-D motion and tunneling of a relativistic particle with almost evident generalization of articles [3], adding only the solution of the Klein paradox and its applications.

2) As to the 2-D and 3-D motion and tunneling of relativistic particles and photons, one can easily generalize [5] [20] in the case of non-relativistic particles and photons.

3) As to the time-energy indeterminacy relations, their derivation for the Klein-Gordon and Dirac cases is an evident generalization of the non-relativistic case.

4) As to the discrete energy spectra for time operator in relativistic case, this problem is also easy to be resolved, evidently generalizing the non-relativistic case.

5) Now the problems of the time analysis of two identical particles (bosons and fermions) tunneling through each other are remained.

6) There are remained the problems of space-time analysis for two- and multi-step nuclear reactions (with direct, pre-compound and compound-nucleus reactions) with thorough investigation of various mechanisms in the center-of-mass and laboratory systems.

7) There are remained many problems of space-time analysis for more complicated nuclear reactions.

8) As a continuation and extension of the first results, exposed in [63]-[65] for the age of stars and planets, a scientific program of the future investigations can be proposed, taking into account.

a) Chains of the successive decays of every star or terrestrial chronometer.

b) The exact evaluations of the Doppler effect during multiple recoils from emitted or absorbed $\gamma$-rays by excited radioactive nuclei.

c) Several initial excited states of every star or terrestrial chronometer.

d) The joint considering the both $\alpha$-and $\beta$-radioactive decays.

e) The necessary modifications of processes of the stellar and the possible cosmic nucleo-synthesis.

\subsubsection{A Curious Remark}

Following [20] [21], one can present here also the next description of the alternative quantum-mechanical approach to time for systems with continuous energy spectra: In the history of time in quantum mechanics it is also known the approach POVM (the positive-operator-value-measure approach), non rarely being used even now (see, in particular, [66]-[72]), rising to the earlier version of quantum measurement theory. This approach is generally known in quantum measurement theory approximately from the sixties and it was used in the simple form as an illustration for describing a free motion still in [73]. Namely in [66]-[72] (sometimes with some simplifications and abbreviations) it was stated that he generalized expansion of unity (or POV measure) is reproduced from any self-adjoint expansion of time operator into the extended Hilbert space (with the negative values of energy over the left semi-axis) by citing the Naimark dilatation theorem [74]. However, in fact it was realized only in the simple cases like free particle motion. The concluding work of this approach [72] is mainly related, generally speaking, to the evidently not completed quantum measurement theory but not to the fundamental quantum theory.

Our approach is based on another Naimark theorem (see reference [23] above) without any extension of the physical Hilbert space with usual wave functions (wave packets) with further back projection on the previous space of wave functions. Moreover, it was published in [3] [19] earlier that articles [66]-[72]. Based on the Naimark theorem [23], published earlier, it much more direct, simple and simultaneously not less rigorous than the approach POVM. And what is more, it is related with the standard fundamental quantum mechanics. Moreover, within the POVM approach nobody constructed time operator for the systems with discrete energy spectra and for quantum field theory.

\section{Final Conclusion}

Here it is shown really that the fundamental quantum mechanics up to last time was not completed, long time 
after the Pauli book [18] time considered as a classic parameter. And only the utilization of the mathematical Naimark theory [23] for the widened mathematical base of quantum mechanics and the construction of time operator for systems with the discrete energy spectra permitted to finally complete the fundamental quantum mechanics and formulate the remain problems in time analysis of tunneling and nuclear processes.

Of course, there are many things to do also in quantum measurement theory and the interpretation of it.

\section{References}

[1] Ginzburg V.L. (1999) What Problems of Physics and Astrophysics Seem Now to Be Especially Important and Interesting (Thirty Years Later, Already on the Verge of XXI Century)? Physics-Uspekhi, 42, 353-373. http://dx.doi.org/10.1070/PU1999v042n04ABEH000562

[2] Einstein A., Podolsky, B. and Rosen, T. (1935) Can Quantum-Mechanical Description of Physical Reality Be Considered Complete? Physical Review, 47, 777-780.

[3] Olkhovsky, V.S., Recami, E. and Jakiel, J. (2004) Unified Time Analysis of Photon and Particle Tunneling. Physics Reports, 398, 133-178. http://dx.doi.org/10.1016/j.physrep.2004.06.001

[4] Olkhovsky, V.S. and Recami, E. (2007) Time as a Quantum Observable. International Journal of Modern Physics A, 22, 5063-5087. http://dx.doi.org/10.1142/S0217751X0703724X

[5] Olkhovsky, V.S. (2009) Time as a Quantum Observable, Canonically Conjugated to Energy, and Foundations of SelfConsistent Time Analysis of Quantum Processes. Advances in Mathematical Physics. 2009, 83 p. http://dx.doi.org/10.1155/2009/859710

[6] Bohm, D. (1952) A Suggested Interpretation of the Quantum Theory in Terms of Hidden Variables. Physical Review, 85, 66-179.

[7] Pais, A. (1979) Einstein and the Quantum Theory. Reviews of Modern Physics, 51, 863-914. http://dx.doi.org/10.1103/RevModPhys.51.863

[8] Popper, K. (1982) A Critical Note on the Greatest Days of Quantum Theory. Foundations of Physics, 12, 971-976. http://dx.doi.org/10.1007/BF01889270

[9] Holland, P.R. (1993) The Quantum Theory of Motion: An Account of the de Broglie-Bohm Causal Interpretation of Quantum Mechanics. Cambridge University Press, Cambridge. http://dx.doi.org/10.1017/CBO9780511622687

[10] Paty, M. (1995) The Nature of Einstein's Objections to the Copenhagen Interpretation of Quantum Mechanics. Foundations of Physics, 25, 183-204. http://dx.doi.org/10.1007/BF02054665

[11] Everett, H. (1957) “Relative State” Formulation of Quantum Mechanics. Review of Modern Physics, 29, 454-462. http://dx.doi.org/10.1103/RevModPhys.29.454

[12] DeWitt, B.S.M. (1970) Quantum Mechanics and Reality. Physics Today, 23, 30-35. http://dx.doi.org/10.1063/1.3022331

[13] Everett, H. (1973) The Theory of the Universal Wave Function. In: DeWitt, B. and Graham, N., Eds., The ManyWorlds Interpretation of Quantum Mechanics, Princeton University Press, Princeton.

[14] Menskii, M.B. (2005) Concept of Consciousness in the Context of Quantum Mechanics. Physics-Uspekhi, 175, 413435.

[15] Zeh, H.D. (1970) On the Interpretation of Measurement in Quantum Theory. Foundations of Physics, 1, 69-76.

[16] Janik, J.A. (1998) Nauka, Religia, Dzieje. IX Seminarium w Castel Andolfo, 5-7 sierpnia 1997, Wydawn. Uniwers. Jagiell., Krako`w, 15-22.

[17] Polkinghorne, J. (1991) Reason and Reality: The Relationship between Science and Theology. Trinity Press International, Philadelphia.

[18] Pauli, W. (1926) Handbuch der Physik. Vol. 5, by Fluegge, S., Ed., Berlin , 60.

[19] Olkhovsky, V.S. (1984) Investrigation of Nuclear Reactions and Decays by Analysis of Their Duration. Soviet Journal of Nuclear Physics, 15, 130-148.

[20] Olkhovsky, V.S. (2011) On Time as a Quantum Observable Canonically Conjugate to Energy. Physics-Uspekhi, 181, 829-835.

[21] Olkhovsky, V.S. (2012) Time as a Quantum Observable Canonically Conjugate to Energy. Time Analysis of Quantum Processes of Tunneling and Collisions (Nuclear Reactions), LAP LAMBERT Academic Publishing, Saarbrücken, 177 p.

[22] Olkhovsky, V.S. and Recami, E. (1968) Space-time Shifts and Cross Sections in Collisions between Relativistic Wave Packets. Nuovo Cim., A53, 610-615. 
[23] Naimark, M.A. (1940) Izvestiya Akademii Nauk SSSR. Seriya Matematicheskaya, 4, 277.

[24] Carleman, T. (1923) Sur les e’quations i’ntegrales a' noyau re’el et syme’trque. Uppsala.

[25] Akhiezer, N.I. and Glazman, I.M. (1981) The Theory of Linear Operators in Hilbert Space. Pitman, Boston.

[26] von Neumann, J. (1932) Mathematischen Grundlagen del Quantum Mechanik. Hizzel, Leipzig.

[27] Stone, M.H. (1930) Linear Transformations in Hilbert Space: III. Operational Methods and Group Theory. Proceedings of the National Academy of Sciences of the United States of America, 16, 172-175.

[28] Haar, D. (1971) Elements of Hamiltonian Mechanics. Oxford.

[29] Judge, D. and Levis, J.L. (1963) On the Commutator ZLZ, $\varphi$ ]. Physics Letters, 5, 190. http://dx.doi.org/10.1016/S0375-9601(63)96306-0

[30] Carruthers, P. and Nieto, M.M. (1968) Phase and Angle Variables in Quantum Mechanics. Review of Modern Physics, 40, 411. http://dx.doi.org/10.1103/RevModPhys.40.411

[31] Davydov, A.S. (1976, 1982) Quantum Mechanics. Pergamon, Oxford.

[32] Olkhovsky, V.S., Maydanyuk, S.P. and Recami, E. (2010) Non-Self-Adjoint Operators as Observables in Quantum Theory and Nuclear Physics. Physics of Particles and Nuclei, 41, 508-530. http://dx.doi.org/10.1134/S1063779610040027

[33] Schweber, S.S. (1961) An Introduction to Relativistic Quantum Field Theory. Row, Peterson and Co., New York.

[34] Holevo A.S. (1982) Probabilistic and Statistical Aspects of Quantum Theory (Amsterdam).

[35] Rosenbaum, D.M. (1969) Super Hilbert Space and the Quantum-Mechanical Time Operators. Journal of Mathematical Physics, 10, 1127.

[36] Fujiwara, I. (1979) Well-Defined Time Operators in Quantum Mechanics. Progress of Theoretical Physics, 62 , 1179.

[37] Goto, T., et al. (1980) The Time as an Observable in Quantum Mechanics. Progress of Theoretical Physics, 64, 1. http://dx.doi.org/10.1143/PTP.64.1

[38] Olkhovsky, V.S. and Romanyuk, M.V. (2009) Particle Tunneling and Scattering in a Three-Dimensional Potential with a Hard Core and an External Potential Barrier. Nuclear Physics and Atomic Energy, 10, 273-281.

[39] Olkhovsky, V.S. and Romanyuk, M.V. (2011) On Two-Dimensional Above-Barrier Penetration and Sub-Barrier Tunneling for Non-Relativistic Particles and Photons. Journal of Modern Physics, 2, 1166-1171.

[40] Olkhovsky, V.S., Dolinska, M.E. and Omelchenko, S.A. (2006) The Possibility of Time Resonance (Explosion) Phenomena in High-Energy Nuclear Reactions. Central European Journal of Physics, 4, 223-240. http://dx.doi.org/10.2478/s11534-006-0008-z

[41] Olkhovsky, V.S., Dolinska, M.E. and Omelchenko, S.A. (2011) On New Experimental Data Manifesting the Time Resonances (or Explosions). Central European Journal of Physics, 9, 1131-1133. http://dx.doi.org/10.2478/s11534-011-0009-4

[42] Olkhovsky, V.S. and Omelchenko, S.A. (2011) On the Space-Time Description of Interference Phenomena in Nuclear Reactions with Three Particles in the Final Channel. The Open Particle and Nuclear Physics Journal, 4, 435-438.

[43] Olkhovsky, V.S. (2011) On the Time Analysis of Nuclear Reactions. Fundamental Journal of Modern Physics, 1, 63-132.

[44] Abolhasani, M. and Golshani, M. (2000) Tunneling Times in the Copenhagen Interpretation of Quantum Mechanics. Physical Review A, 62, Article ID: 012106. http://dx.doi.org/10.1103/PhysRevA.62.012106

[45] Cardone, F., Maydanyuk, S.P., Mignani, R. and Olkhovsky, V.S. (2006) Multiple Internal Reflections during Particle and Photon Tunneling. Foundations of Physics Letters, 19, 441-452. http://dx.doi.org/10.1007/s10702-006-0903-y

[46] Enders, A. and Nimtz, G. (1992) On Superluminal Barrier Traversal. Journal de Physique I, 2, 1693-1708.

[47] Enders, A. and Nimtz, G. (1993) Photonic Tunneling Times. Journal de Physique I, 3, 1089-1098.

[48] Enders, A. and Nimtz, G. (1993) Propagation of an Electromagnetic Pulse through a Waveguide with a Barrier. Physical Review, B47, 19605-19618.

[49] Enders, A. and Nimtz, G. (1993) Evanescent-Mode Propagation and Quantum Tunneling. Physical Review, E48, 632-643.

[50] Nimtz, G. (1997) Tunneling and Its Applications. World Science, Singapore City, 223-237.

[51] Steinberg, A.M., Kwiat, P.G. and Chiao, R.Y. (1993) Measurement of the Single-Photon Tunneling Time. Physical Review Letters, 71, 708-711. http://dx.doi.org/10.1103/PhysRevLett.71.708

[52] Chiao, R.Y. and Steinberg, A.M. (1997) Progress in Optics. Vol. 37, Elsevier Science, Amsterdam, 346-405.

[53] Longhi, S., Laporta, P., Belmonte, M. and Recami, E. (2002) Measurement of Superluminal Optical Tunneling Times 
in Double-Barrier Photonic Band Gaps. Physical Review E, 65, Article ID: 046610. http://dx.doi.org/10.1103/PhysRevE.65.046610

[54] Olkhovsky, V.S., Petrillo, V. and Zaichenko, A.K. (2004) Decrease of the Tunneling Time and Violation of the Hartman Effect for Large Barriers. Physical Review A, 70, Article ID: 034103. http://dx.doi.org/10.1103/PhysRevA.70.034103

[55] Chew, H., Wang, D.S. and Kerker, M. (1979) Elastic Scattering of Evanescent Electromagnetic Waves. Applied Optics, 18, 2679-2677. http://dx.doi.org/10.1364/AO.18.002679

[56] Chandiramani, K.L.J. (1974) Diffraction of Evanescent Waves, with Applications to Aerodynamically Scattered Sound and Radiation from Unbaffled Plates. Journal of the Acoustical Society of America, 55, 19-25. http://dx.doi.org/10.1121/1.1919471

[57] Eremin, N.V., Omelchenko, S.A., Olkhovsky, V.S., et al. (1994) Temporal Description of Interference Phenomena in Nuclear Reactions with Two-Particle Channels. Modern Physics Letters, 9, 22849-2856.

[58] Olkhovsky, V.S., Dolinska, M.E. and Omelchenko, S.A. (2011) On Scattering Cross Sections and Durations Near an Isolated Compound-Resonance, Distorted by the Non-Resonant Background, in the Center-of-Mass and Laboratory Systems. Applied Physics Letters, 99, Article ID: 244103.

[59] Olkhovsky, V.S., Doroshko, N.L. and Lokotko, T.I. (2013) On the Cross Section and Duration of the Neutron-Nucleus Scattering with Two Overlapped Resonances in the Center-of-Mass System and Laboratory System. Proceedings of the 4th International Conference on Current Problems in Nuclear Physics and Atomic Energy (NPAE-2012), Kyiv, 3-7 September 2012, 192-197.

[60] Olkhovsky, V.S., Dolinska, M.E. and Omelchenko, S.A. (2013) On the Cross Section and Duration of the Neutron-Nucleus Scattering with a Resonance, Distorted by a Non-Resonant Background, in the Center-of-Mass System and Laboratory System. Proceedings of the 4th International Conference on Current Problems in Nuclear Physics and Atomic Energy (NPAE-2012), Kyiv, 3-7 September 2012, 198-201.

[61] Olkhovsky V.S., Dolinska M.E. and Omelchenko S.A. (2006) The Possibility of Time Resonance (Explosion) Phenomena in High-Energy Nuclear Reactions. Central European Journal of Physics, 4, 1-18.

[62] Olkhovsky, V.S. and Omelchenko, S.A. (2011) On the Space-Time Description of Interference Phenomena in Nuclear Reactions with Three Particles in the Final Channel. The Open Particle and Nuclear Physics Journal, 4, 35-38.

[63] Olkhovsky, V.S. and Dolinska, M.E. (2010) Вісник Київського університету. Серія: Фіз.-мат. науки, вип.3, 82-86 [The Proceedings of International Conference "Humboldt Cosmos: Science and Society”, HCS2 Kiev2009].

[64] Olkhovsky, V.S. and Dolinska, M.E. (2010) On the Modification of Methods of Nuclear Chronometry in Astrophysics and Geophysics. Centr. Europ. J. Phys., 8, 95-100.

[65] Olkhovsky, V.S. (2014) On the Modification of Nuclear Chronometry in Astrophysics and Geophysics. Development in Earth Science (DES), 2, 1-7.

[66] Kobe, D.H. and Aguilera-Navarro, V.C. (1994) Derivation of the Energy-Time Uncertainty Relation. Physical Review A, 50, 933-941. http://dx.doi.org/10.1103/PhysRevA.50.933

[67] Grot, N., Rovelli, C. and Tate, R.S. (1996) Time of Arrival in Quantum Mechanics. Physical Review A, 54, $4676-4687$. http://dx.doi.org/10.1103/PhysRevA.54.4676

[68] Leo’n, J. (1997) Time Operator in the Positive-Operator-Value-Measure Approach. Journal of Physics A: Mathematical and General, 30, 4791-4799.

[69] Aharonov, Y., Oppenheim, J., Popescu, S., Reznik, B. and Unruh, W.G. (1998) Measurement of Time of Arrival in Quantum Mechanics. Physical Review A, 57, 4130-4142. http://dx.doi.org/10.1103/PhysRevA.57.4130

[70] Muga, J., Papao, J. and Leavens, C. (1999) Arrival Time Distributions and Perfect Absorption in Classical and Quantum Mechanics. Physics Letters A, 253, 21-27. http://dx.doi.org/10.1016/S0375-9601(99)00020-1

[71] Muga, J., Egusquiza, I.L., Damborenea, J.A. and Delgado, F. (2002) Bounds and Enhancements for Negative Scattering Time Delays. Physical Review A, 66, Article ID: 042115. http://dx.doi.org/10.1103/PhysRevA.66.042115

[72] Muga, J.G., Mayato, R.S. and Egusquiza, I.L. (2007) Time in Quantum Mechanics-Lecture Notes in Physics, v.1, 2007, v.2, 2009, Springer, Berlin.

[73] Aharonov, Y. and Bohm, D. (1961) Time in the Quantum Theory and the Uncertainty Relation for Time and Energy. Physical Review, 122, 1649-1657. http://dx.doi.org/10.1103/PhysRev.122.1649

[74] Naimark, M.A. (1943) Izv.AN SSSR. Seria Matematicheskaya, 7, 237. 